\title{
Spinar model for the fast-rotating collapsing objects
}

\author{
Chasovnikov Aristarh ${ }^{\mathbf{1}}$ \\ Lomonosov Moscow State University \\ Leninskie Gory, Moscow, 119991, Russian Federation \\ E-mail: chasovnikovesai.msu.ru
}

\section{Lipunov V. M}

Lomonosov Moscow State University

Leninskie Gory, Moscow, 119991, Russian Federation

E-mail: Iipunov2007@gmail.com

\section{Gorbovskoy E.S}

Lomonosov Moscow State University

Leninskie Gory, Moscow, 119991, Russian Federation

E-mail: gorbovskoyegmail.com

\section{Lipunova G. V}

Lomonosov Moscow State University

Leninskie Gory, Moscow, 119991, Russian Federation

E-mail: gvlipunova@gmail.com

\begin{abstract}
After observing the kilonova in the optical range, one of the most interesting tasks of astronomy is to observe a short gamma-ray burst that can be formed when neutron stars merge. Using the spinar paradigm, we model this process to evaluate the possibility of such observations by a network of robotic optical telescopes MASTER. We show that, despite the obvious complexity of such observations, the MASTER may be able to make them.
\end{abstract}

The Golden Age of Cataclysmic Variables and Related Objects V (GOLDEN2019)

2-7 September 2019

Palermo, Italy

\footnotetext{
${ }^{1}$ Speaker

(C) Copyright owned by the author(s) under the terms of the Creative Commons 


\section{Introduction}

In August 2017, the gravitational-wave detectors LIGO and VIRGO first registered the merger of neutron stars[3]. The optical counterpart of this event, the kilonova, was independently detected by the $1 \mathrm{~m} 2 \mathrm{~h}$ Swope, DLT 40, VISTA, MASTER, DECam, and Las Cumbres telescopes[5][3]. The search for such optical transients is one of the most important tasks of modern astronomy. In addition to being interesting by themselves, such objects can provide valuable information about the structure of the Universe and put new restrictions on cosmological models. However, the kilonova is not the only manifestation of neutron star mergers in electromagnetic radiation. At the moment of merging, a short gamma-ray burst can be observed, as well as powerful radiation in all other ranges, including in optics. Observing this phenomenon is a very difficult task, since its duration is extremely short. However, we hope that such observations will become possible in the near future. In this work, we estimate the duration of the merger, as well as the luminosity in the optical and radio bands. Since our task is to approximate the results, we used a one-dimensional spinar model, which, although high-quality, takes into account all the main effects that occur during the merge stage.

\section{Model}

\subsection{Spinar}

A spinar is a quasi-equilibrium object in which two main opposing forces operate: gravitational and centrifugal[6]. By adding some additional forces to this model, we apply it to simulate the fusion process of neutron stars.

The spinar model was used to calculate various processes. It can be used to explain such phenomena as, for example, the x-ray plateau in gamma-ray burst [7]. In the following works, the spinar model was used to explain all the characteristic elements of the gamma-ray burst brightness curve[6]. This model can also explain the precursor and afterglow. Despite this, for a long time it was believed that when neutron stars merge, spinar will not have time to form.

Modern three-dimensional calculations show that when neutron stars merge, an object is formed that cannot instantly collapse into a black hole, even if the total mass of the components of the binary system significantly exceeds the Oppenheimer-Volkov limit[13][17]. An observational confirmation of the formation of a spinar during a merger is the afterglow observed after the GW170817 event by the FERMI space telescope[15].

We model the merging process of neutron stars starting from the moment when the major half-axis of the neutron star orbit is $150 \mathrm{~km}$.the Input data of our model are the masses of neutron stars and the initial magnetic fields. We calculate the radii of neutron stars using a qualitative equation of state, which is chosen so that the Oppenheimer-Volkov limit is 2.5 times the mass of the Sun. In the first stage, we calculate the convergence process caused by the radiation of gravitational waves. During this approach, the electromagnetic radiation in this system, which occurs due to the presence of a quadrupole component, begins to increase sharply. We believe that the radiation at this moment is quite close to isotropic.

The second stage in our model is the spinar stage. At this time, a jet is formed in the system, and the main radiation goes into it and can be observed as a short gamma-ray burst.

A separate computational task in our work was to ensure the smoothest possible transition from the first stage to the second. The cross-linking of these models was carried out in such a way that the density and moment of inertia of the system did not undergo a jump, and the other parameters changed as smoothly as possible. 
Despite the fact that the lifetime of a spinar, as well as its luminosity, strongly depends on the magnetic field, the final fate of an object depends only on the ratio between its mass and the Oppenheimer-Volkov limit.

If the mass is greater than the Oppenheimer-Volkov limit, it collapses into a black hole; if it is smaller, a Magnetar is formed.

In order for the spinar model to be applied to neutron stars, we added two additional forces besides gravitational and centrifugal: the pressure of nuclear matter and the dissipative force. Spinar rotation is slowed down by a magnetic field.

When calculating the forces mentioned above, we took into account the Lenz-Thirring effect, the black hole hairless theorem, and the operation of the Dynamo mechanism due to differential rotation.

\subsection{A qualitative equation of state for a non-rotating non-magnetized neutron star}

So, we present the equation of state for spinar:

$$
\frac{d^{2} R}{d^{2} t}=F_{g}+F_{n}+F_{c}+F_{d}
$$

where $\mathrm{R}$ is the spinar radius, $F_{g}$ is the gravitational force, $F_{n}$ is the nuclear force, $F_{c}$ is the centrifugal force, and $F_{d}$ is the dissipative force. Naturally, we are talking about specific forces acting on a unit of mass[7].

The gravitational force is calculated taking into account the main effects of General relativity[14].

$$
F_{g}=-\frac{G M}{x^{3}} \frac{\left(x^{2}-2 a \sqrt{x}+a^{2}\right)^{2}}{(\sqrt{x}(x-2)+a)^{2}},
$$

Here:

$$
a=\frac{I \omega c}{G M^{2}}
$$

is the Kerr parameter, $I$ - the moment of inertia, $\omega$ - the speed of rotation, $M$ - the mass of the spinar,

$R_{g^{-}}$gravitational radius.

$$
x=\frac{2 R}{R_{g}}
$$

Centrifugal force:

$$
F_{c}=\omega^{2} R
$$

The nuclear pressure is calculated using a relativistic invariant:

$$
\begin{gathered}
P=\rho \sqrt{c^{4}+b \rho^{2 / 3}+(Q / M)^{2}}-c^{2} \\
F_{n}=\frac{P}{\rho R}
\end{gathered}
$$

Here $\rho$ is the spinar density, $Q$ is the thermal energy.

In spinar, of course, there are dissipative forces at work. They are caused by the presence of a magnetic field and viscosity in neutron stars. However, the viscosity depends on the equation of state, which is very poorly known, so the detailed calculation of dissipative forces is very complex and inaccurate. We are not trying to calculate the viscosity in the matter of a neutron star, because we need a magnetic field to simulate the action of these forces. Since the amount of dissipative forces has very little effect on the collapse process, we use a simple qualitative formula

Here $\chi=0,04$.

$$
F_{d}=-\frac{\omega}{2 \pi \chi} \frac{d R}{d t}
$$

\subsection{Calculating the radius of neutron stars}


To calculate the radii of neutron stars, we used the same equation of state, assuming that the orbital rotation speed is much higher than the individual rotation speeds of neutron stars. Thus,

$$
\begin{gathered}
F_{c}=0 ; F_{d}=0 \\
0=F_{g}+F_{n}
\end{gathered}
$$

\subsection{Slowing down spinar rotation}

We believe that the density of a neutron star is weakly dependent on the radius, so the moment of inertia

$$
I \approx 2 / 5^{M} R^{2}
$$

Accordingly, the moment of impulse

$$
J=I \omega
$$

The change of the angular momentum is calculated according to the magnetodipole formula:

$$
\frac{d J}{d t}=-\frac{\mu^{2} \omega^{2}}{G M}
$$

Taking into account relativistic effects, the dipole moment does not remain constant, but tends to zero when the object approaches the gravitational radius. It can be approximately calculated as follows[1]:

Here:

$$
\mu=\mu_{0}\left(\frac{R_{0}}{R}\right)^{2} \frac{\xi\left(x_{0}\right)}{\xi(x)}
$$

$$
\xi(x)=\frac{1}{x}+\frac{1}{2 x^{2}}+\ln \left(1-\frac{1}{x}\right)
$$

\section{$2.5 \quad$ Full luminosity}

To calculate the luminosity before the merger, we use the magnetic quadrupole approximation Then

where $\dddot{D}=\omega_{o r b}^{3} a \mu, a$ - orbital radius.

$$
L=\frac{\dddot{D}^{2}}{180 c^{5}}
$$

After the merger, the magnetic-dipole radiation is calculated according to the formula:

where

$$
L=\alpha^{2} \frac{\mu^{2} \omega}{R^{3}}
$$

$$
\alpha=\sqrt{\frac{x^{2}+a^{2}-2 x}{x^{2}+a^{2}}} .
$$

Here, $\alpha$ is the time dilatation function[19].

\subsection{Spectrum}

Unfortunately, our calculations do not give an idea of the spectrum of radiated energy. Since we give a rough qualitative estimate of luminosity, we use the spectrum of the radio pulsar in the crab nebula PSR B0531+21 to calculate the distribution of radiated energy over the spectrum. 


\section{Results}

The main task of our calculations was a qualitative assessment of the integral luminosity of the spinar during the collapse, as well as the absolute stellar magnitude in the optical range and the flux in the radio range. The figures show graphs of the dependence of these values on time.

As you can see from the pictures, the merging process is very fast, but its luminosity is quite high. Random observation of such flashes in the optical range is almost impossible. However, LIGO and Virgo are able to pick up a signal from a neutron star merger a few tens of seconds before the merger begins. MASTER is a mobile system and can be set up during this time. Therefore, the observation of such phenomena may become possible in the near future.

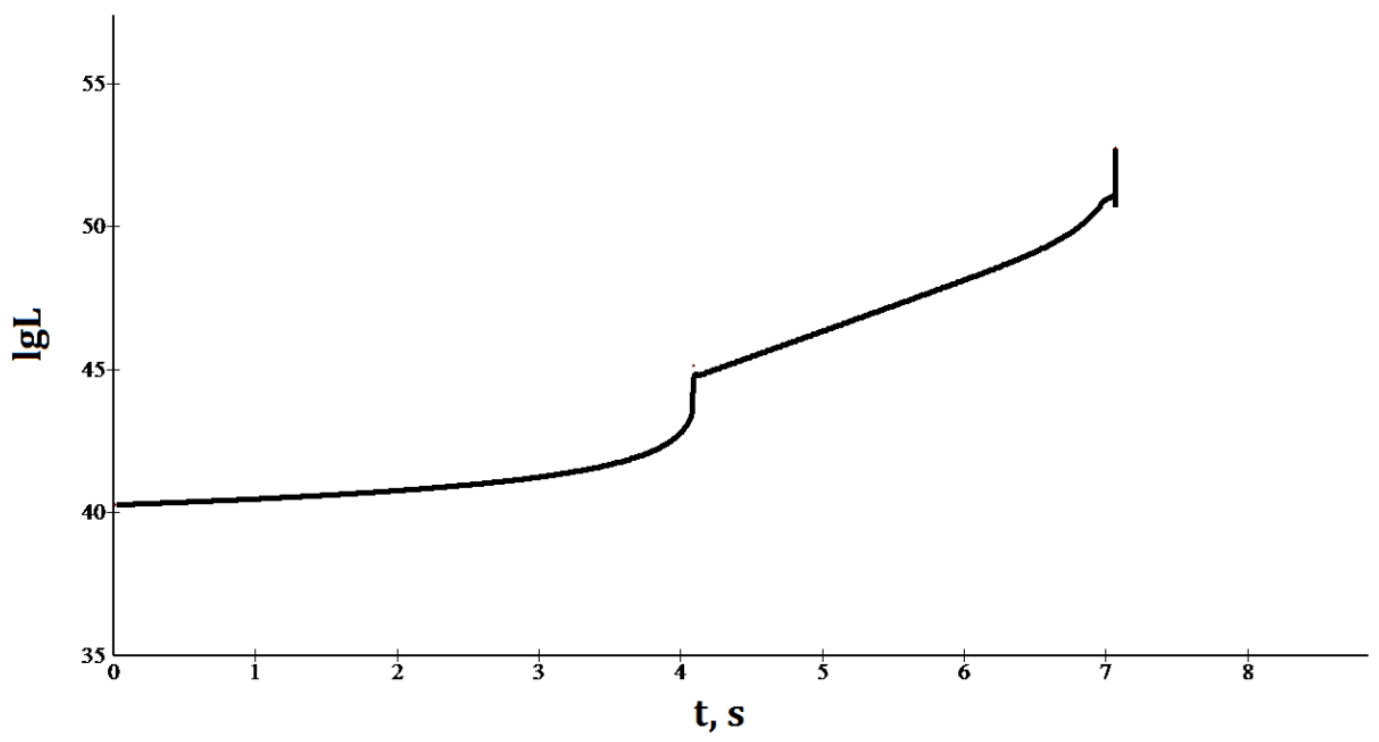

Fig 1. Full luminosity. $M>$ Mov

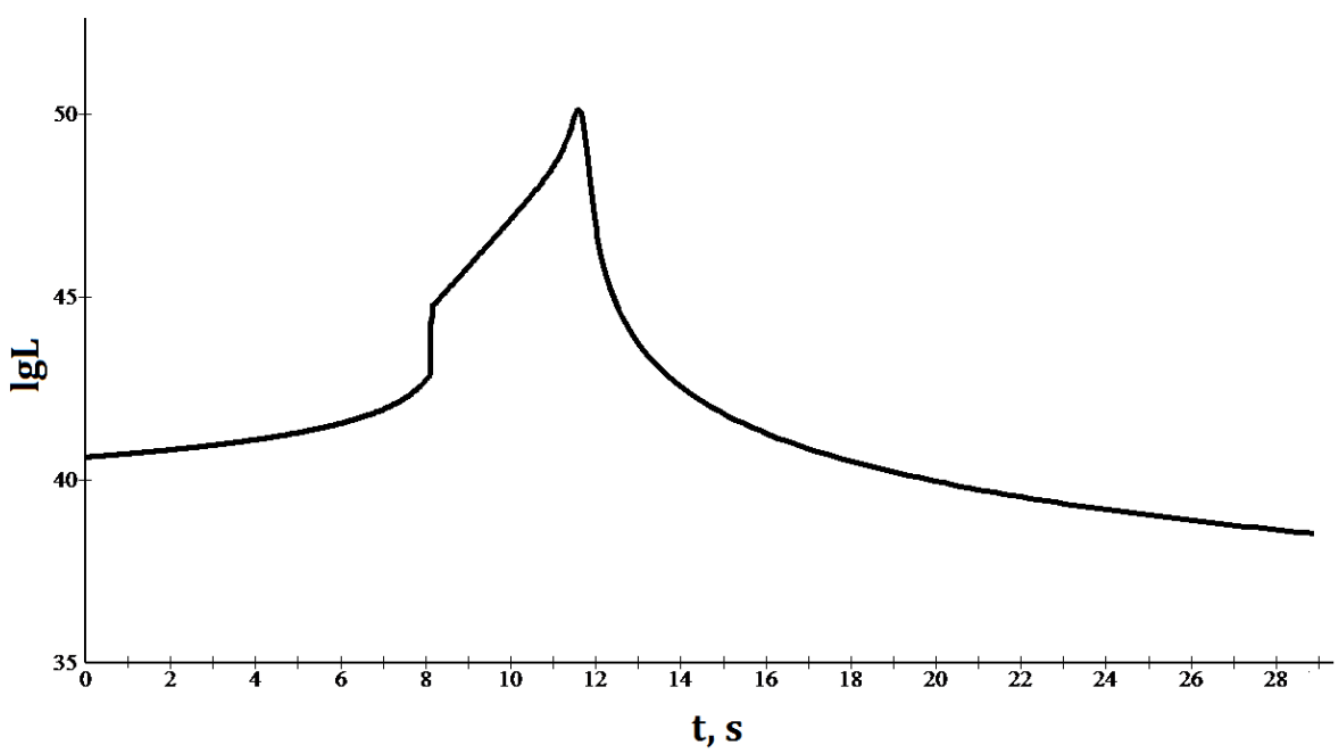

Fig 2. Full luminocity. $M<$ Mov 


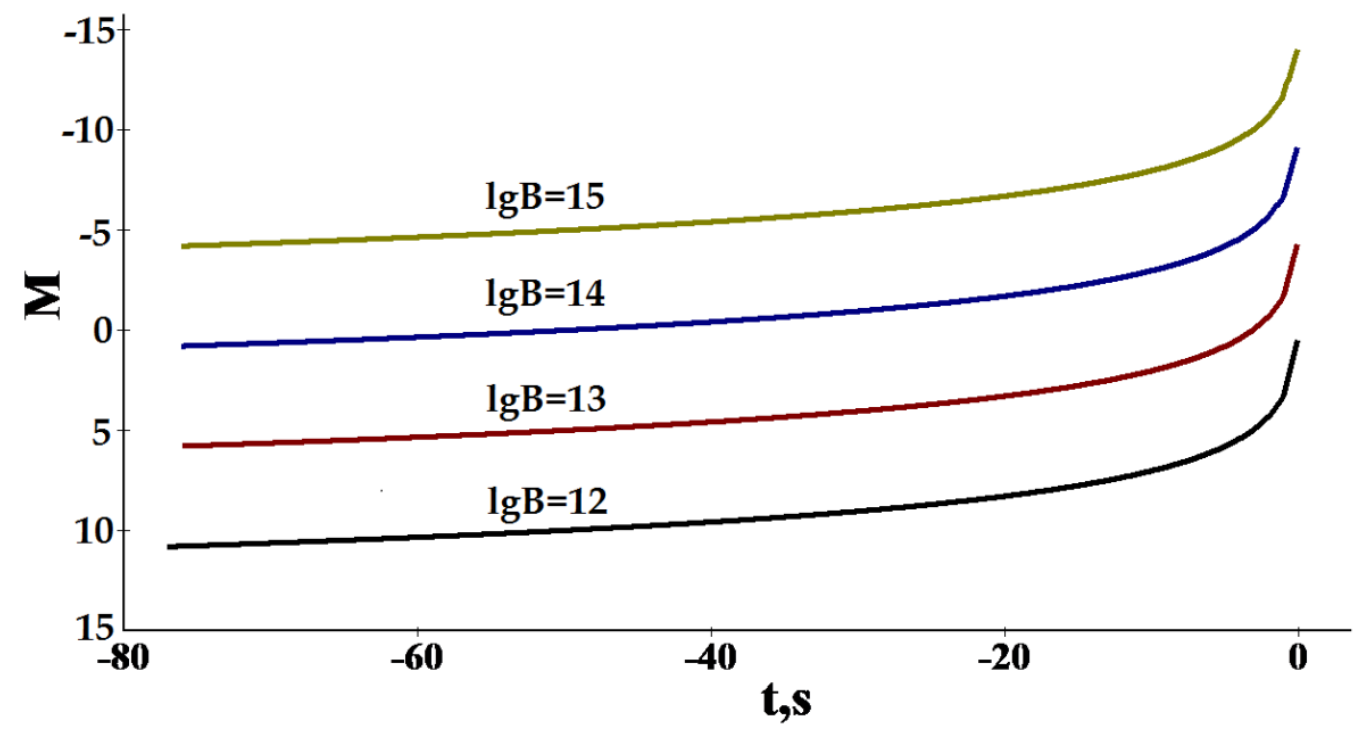

Fig 3. Absolute magnitude. $M=4 M o$

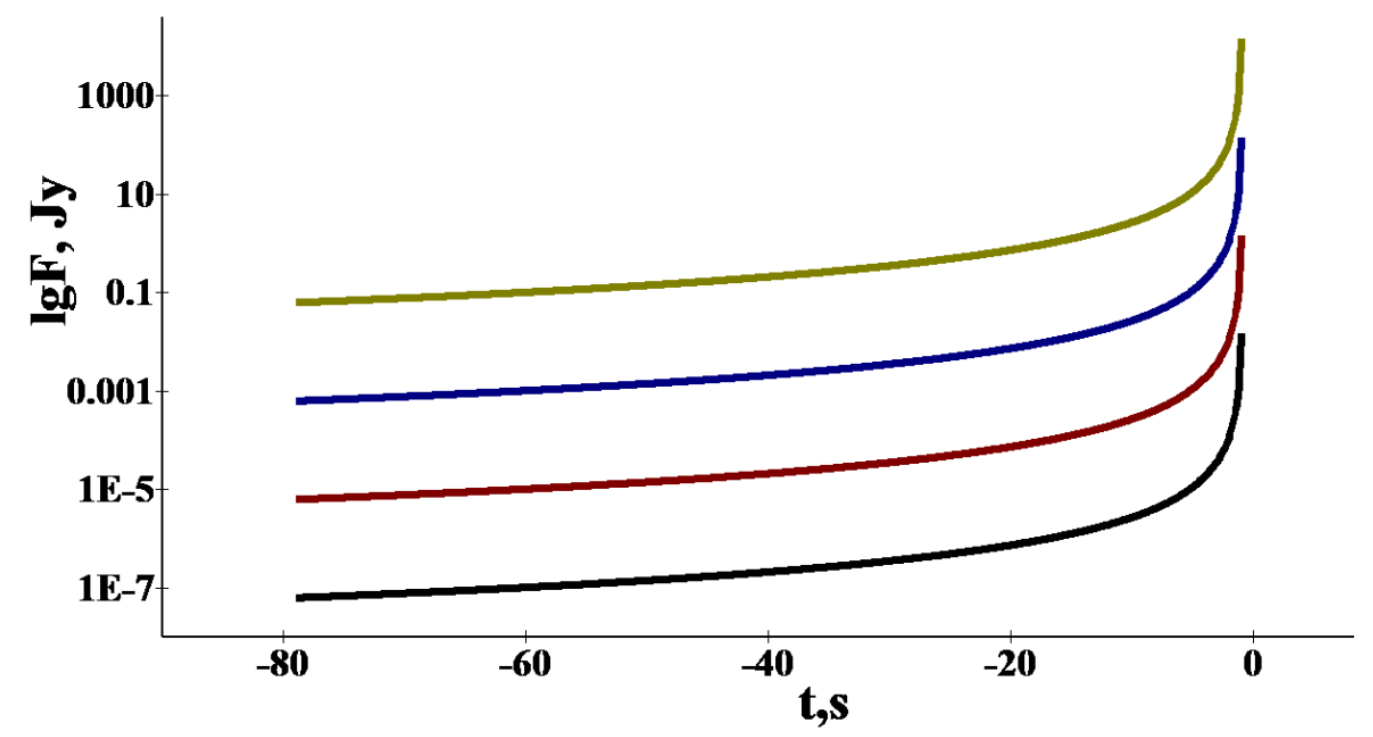

Fig 4. Flux density in the radio band. Distance - 100Mpc. $M=4 M o$

\section{Acknowledgments}

VL is supported by the BRICS RFBR grant 17-52-80133, A.Ch. is supported by RFBR grant 19-29-11011

\section{References}

[1] Ginsburg V. L., Ozernoy L. M., About the gravitational collapse of a magnetic star. 1964, Sov. Phys. JEPT, 47,1030

[2] LIGO Scientific Collaboration and Virgo Collaboration. Multi-messenger Observations of a Binary Neutron Star Merger. The Astrophysical Journal Letters, 848:L12 (59pp), 2017 October 20

[3] Lipunov et al., 2010, MASTER Robotic Net, Advances in Astronomy, vol. 2010, p.30L 
[4] Lipunov, V. M.; Panchenko, I. E. Pulsars revived by gravitational waves. Astronomy and Astrophysics, v.312, p.937-940. August 1996

[5] Lipunov V. M. et al. MASTER Optical Detection of the First LIGO/Virgo Neutron Star Binary Merger GW170817. The Astrophysical Journal Letters, 850:L1 ( 9pp) , 2017 November 20

[6] Lipunov V. M. and E. Gorbovskoy. Spinar paradigm and the central engine of gamma-ray bursts. Mon. Not. R. Astron. Soc.383, 1397-1412 (2008)

[7] Lipunov V. M and Gorbovskoy E. S. AN EXTRA LONG X-RAY PLATEAU IN A GAMMA-RAY BURST AND THE SPINAR PARADIGM. The Astrophysical Journal, 665:L97-L100, 2007 August 20

[8] Lipunov V. M. et al. The Discovery of the neutron stars merger GW1 70817/GRB170817A and a Binary Neutron Stars Evolution. New Astronomy, Volume 63, p. 48-60. August 2018

[9] Lipunov V. M. et al. Evolution of the double neutron star merging rate and the cosmological origin of gammaray burst sources. Astrophysical Journal v.454, p.593. December 1995

[10] Lipunova G. V et al. Population synthesis of gamma-ray bursts with precursor activity and the spinar paradigm. Mon. Not. R. Astron. Soc.397, 1695-1704. 2009

[11] Lipunova G. V., 1997, Astron. Lett., 23, 84

[12] Lipunova G. V., Lipunov V. M., 1998, A\&A, 329, L29

[13] Lunan Sun, Milton Ruiz and Stuart L. Shapiro. Magnetic Braking and Damping of Differential Rotation in Massive Stars. Physical Review D, Volume 99, Issue 6, id.064057. March 2019

[14] Banibrata Mukhopadhyay. DESCRIPTION OF PSEUDO-NEWTONIAN POTENTIAL FOR THE RELATIVISTIC ACCRETION DISK AROUND KERR BLACK HOLES. The Astrophysical Journal, Volume 581, Issue 1, pp. 427-430. December 2002

[15] Van Putten, Maurice H. P. M.; Della Valle, Massimo. Observational evidence for Extended Emission to GW170817. Monthly Notices of the Royal Astronomical Society: Letters, Volume 482, Issue 1, p.L46-L49. January 2019

[16] Ashok Razdan. Crab pulsar spectrum: a non-extensive approach. New Astronomy, Volume 37, p. 5-8. May 2015

[17] Ruiz, Milton; Tsokaros, Antonios; Paschalidis, Vasileios; Shapiro, Stuart L. Effects of spin on magnetized binary neutron star mergers and jet launching. Physical Review D, Volume 99, Issue 8, id.084032. April 2019

[18] Stratta, Giulia; Dainotti, Maria Giovanna; Dall'Osso, Simone; Hernandez, X.; De Cesare, On the magnetar origin of the GRBs presenting X-ray afterglow plateaus. GiovanniThe Astrophysical Journal, Volume 869, Issue 2, article id. 155,10 pp. December 2018About the gravitational collapse of a magnetic

[19] Thorne K. S., Price R. H., Macdonald D. A., 1986, Black Holes: The Membrane Paradigm. Yale Univ. Press, New Haven 